\title{
RAISING THE PROFILE OF ADOLESCENT NUTRITION IN PAKISTAN: LEARNINGS ON THE JOURNEY FROM POLICY TO ACTION
}

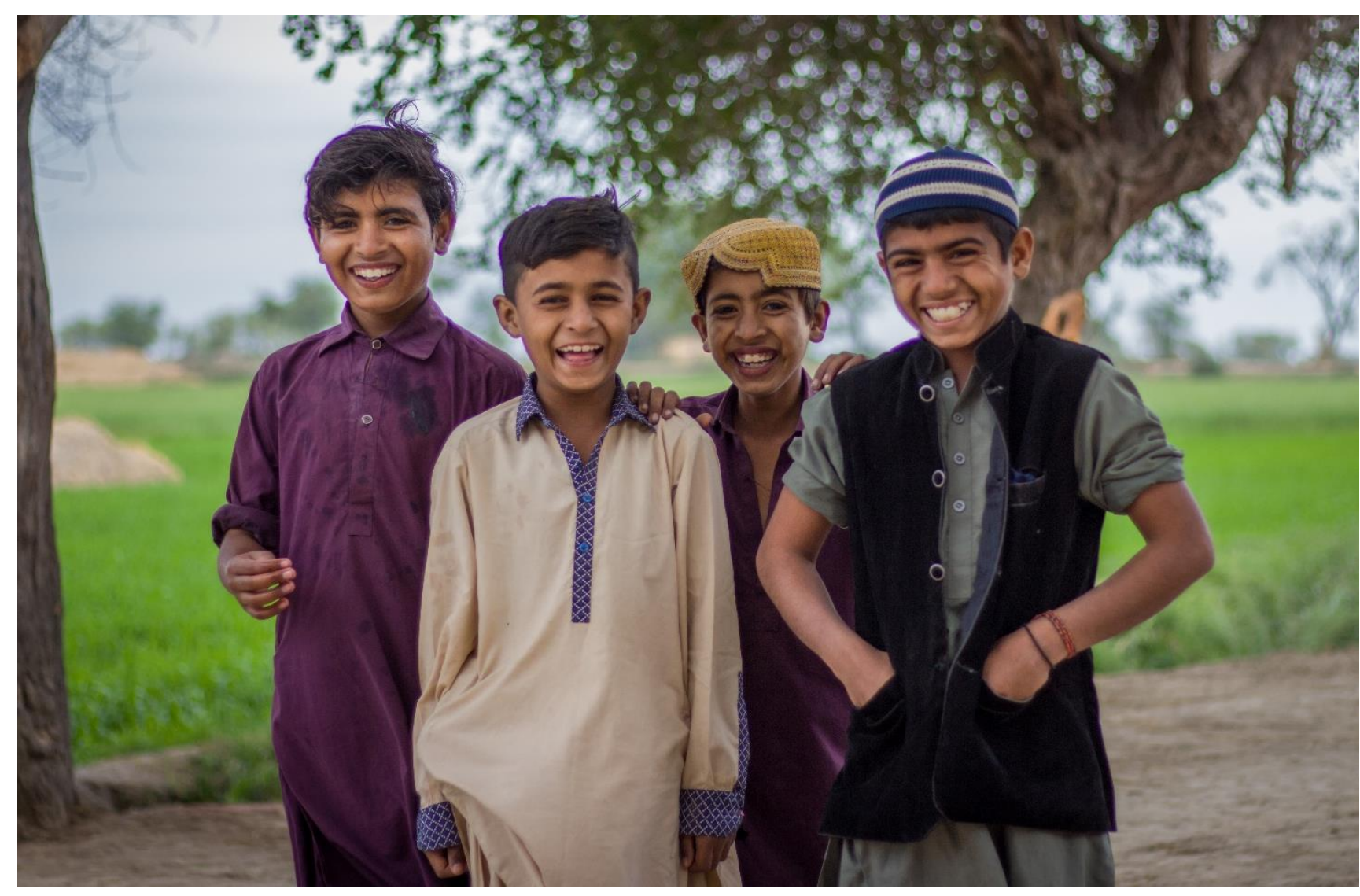

GAIN Working Paper n¹8

March, 2021

The GAIN Pakistan Adolescent Nutrition Project Team 


\section{ABOUT GAIN}

The Global Alliance for Improved Nutrition (GAIN) is a Swiss-based foundation launched at the UN in 2002 to tackle the human suffering caused by malnutrition. Working with governments, businesses and civil society, we aim to transform food systems so that they deliver more nutritious food for all people, especially the most vulnerable.

\section{Recommended citation}

The Global Alliance for Improved Nutrition (GAIN) Adolescent Nutrition Project Team. Raising the profile of adolescent nutrition in Pakistan: learnings on the journey from policy to action. Global Alliance for Improved Nutrition (GAIN). Working Paper \#18. Geneva, Switzerland, 2021. DOI: https://doi.org/10.36072/wp.18

\section{(C) The Global Alliance for Improved Nutrition (GAIN)}

This work is available under the Creative Commons Attribution-Non-Commercial-Share Alike 4.0 IGO licence (CC BY-NC-SA 4.0 IGO; https://creativecommons.org/licenses/by-nc-sa/4.0/). Under the terms of this licence, you may copy, redistribute and adapt the work for non-commercial purposes, provided the work is appropriately cited, as indicated below. In any use of this work, there should be no suggestion that GAIN endorses any specific organisation, products or services. The use of the GAIN logo is not permitted. If you adapt the work, then you must license your work under the same or equivalent Creative Commons license. The contribution of third parties do not necessarily represent the view or opinion of GAIN.

\section{Acknowledgements}

This paper was a collective effort from the GAIN Pakistan Adolescent Nutrition Project team and consultants. Thanks to our consultants, Niaz Ullah Khan, Manahal Fatima and Fauzia Waqar, for drafting content for the paper and conducting interviews. From GAIN's programmatic team, we would like to thank Zineb Felix, Faiz Rasool, Farrah Naz and Asma Badar for being instrumental in the journey and for contributing valuable insights. Thanks to GAIN's Knowledge Leadership team members, Wendy Gonzalez and Stella Nordhagen, for being generous with their time and technical support to improve the paper. Thanks to Alia Poonawala and Abby Falla from GAIN for the diligence in guiding, developing and finalising this paper. We gratefully acknowledge the financial support of the Dutch Ministry of Foreign Affairs for this project. Finally, to the Government of Pakistan and our partners in country for their commitment to improving adolescent nutrition.

All photographs included in this document have been taken with consent for use in publications.

\section{GAIN WORKING PAPER SERIES}

The GAIN Working Paper Series provides informative updates on programme approaches, research and evaluations, and on topics of relevance for our work.

The Global Alliance for Improved Nutrition (GAIN)

Rue de Varembé 7

1202 Geneva

Switzerland

T: +41 227491850

E: info@gainhealth.org

www.gainhealth.org 


\section{SUMMARY}

Pakistan's adolescent population (circa. 40 million people) is experiencing a double burden of malnutrition, with $21 \%$ of boys and $12 \%$ of girls underweight and $18 \%$ of boys and $17 \%$ of girls overweight or obese. This merits a call to action to prioritise public funding and programming to address the determinants of adolescent malnutrition. Fortunately, the Pakistan Adolescent Nutrition Strategy (PANS), launched in October 2020, is an inspiring example of how political will can translate into effective policy and operational planning and create a framework through which health and social system actors can understand the role they play in preventing and treating adolescent malnutrition. Prior to the launch of PANS, the Government of Pakistan and its partners, including the Global Alliance for Improved Nutrition (GAIN), worked to better understand the status of adolescents' nutritional status, raise the profile of adolescent nutrition in Pakistan and stimulate action on the topic. This paper seeks to provide an overview of the enabling environment milestones on the journey to PANS. It seeks to aid in implementation of PANS by applying the Innocenti Framework on Food Systems for Children and Adolescents to examine certain determinants of adolescent diets, specifically trends in consumption and food behaviours and motivations of adolescents. The analysis makes clear that there has been significant progress in creating an enabling environment for adolescent nutrition and addressing fundamental data gaps, including collecting the first nationally representative data on adolescent nutrition. These findings can further inform research and programmes, fostering wider cross-system collaboration under which food system actors recognise their potential in shaping a food system that works for adolescents.

\section{KEY MESSAGES}

- Among Pakistan's 40 million adolescents, $21 \%$ of boys and $12 \%$ of girls underweight and $18 \%$ of boys and $17 \%$ of girls overweight or obese.

- This is partly because carbonated soft drinks and snacks laden with salt, fat and sugar are very popular with adolescents in Pakistan - across all socio-economic classes, both genders and in urban and (to a lesser extent) rural areas.

- Pakistan requires very little labelling on the composition and nutritional profile of foods; this must be improved, and marketing to adolescents should be curbed.

- Pakistan's Adolescent Nutrition Strategy, a joint undertaking by the Government of Pakistan, UNICEF, The World Health Organization (WHO) and the Global Alliance for Improved Nutrition (GAIN), is an example of how political will can translate into effective policy and operational planning.

- A strong enabling environment for adolescent nutrition has been built across the health, education, and social protection systems in Pakistan.

- Food system interventions and actors have a critical role in tackling the double burden of malnutrition among Pakistan's adolescents. 


\section{BACKGROUND AND OBJECTIVE}

There are approximately 1.2 billion adolescents (young people aged 10-19 years) worldwide, representing the largest generation in history (1). Around $90 \%$ of these adolescents reside in low- and middle-income countries (LMICs). In Pakistan, almost one quarter of the population, around 40 million, are 10-19-year-olds (2). In addition to these large numbers, adolescence merits additional attention because it is a period of significant biological, cognitive, psychological, and emotional development (3). It is also a time for gaining independence, acquiring knowledge, and developing skills, behaviours, and habits that persist into adulthood. Pakistani adolescents' potential, however, is currently constrained by malnutrition: Pakistan's young population faces nutritional deficiencies and the double burden of under- and overnutrition.

Adolescents who endure malnutrition beyond childhood suffer poor health and higher morbidity rates in later life stages (5). With about $15-20 \%$ of total stature and $45 \%$ of adult bone mass achieved during adolescence, it is a critical window for impacting adult height, reducing nutritional deficiencies, and building healthy eating practices $(4,5)$. Poor nutrition during adolescence can also result in an intergenerational cycle of malnutrition and can negate the cumulative benefits of good nutrition accrued in infancy and early life (6, 7). Malnourished adolescents face additional hurdles in education and effective participation in social and economic activities. Investing in adolescent malnutrition is thus investing in economic growth $(6,8)$.

Improving the nutritional status of adolescents can also contribute to the prevention and control of non-communicable diseases (NCDs) (8). High intake of highly processed foods and a high Body Mass Index are key risk factors for NCDs in later life stages. Specifically, frequent consumption of highly processed, energy-dense 'fast foods', high in sugar, salt, and unhealthy fats, especially when it occurs at the expense of more nutritious alternatives, can lead to micronutrient deficiencies, high blood pressure and other acute health issues (1). However, little research has been conducted to understand the preferences and behaviour patterns of Pakistani youth towards unhealthy food consumption, and important data gaps exist in the understanding of dietary practices among adolescents in Pakistan $(9,10)$.

Whilst adolescents are underrepresented in national data on nutritional determinants and outcomes, small studies highlight that there are several drivers of poor adolescent nutrition in Pakistan: poverty, low educational attainment, lack of nutritional knowledge, gender norms, unsafe and unhygienic food, and unequal distribution of food, alongside limited access to highquality health services (9-12). Household food insecurity is another important driver of poor diets amongst adolescents in Pakistan, especially in rural households, more than half of which are food insecure for most of the year (13).

The silent nature of nutritional problems, financial constraints, and an historic lack of data on adolescents' nutritional status, dietary practices, and levels of physical activity have made it challenging to develop appropriate adolescent-specific nutrition strategies and to track progress over time. However, global attention has recently turned to supporting countries to better address the dietary needs of adolescents, including by providing improved governance 
and policy frameworks. For example, the Sustainable Development Goals and the Global Strategy for Women's, Children's, and Adolescent's Health both prioritise adolescent health (13). In 2017, the World Health Organization (WHO) developed and shared the Global Accelerated Action for the Health of Adolescents (AA-HA!) framework. Pakistan was the second country in the Eastern Mediterranean WHO Region to start working on a country contextualisation of the AA-HA! framework. The Government of Pakistan and its partners, including the Global Alliance for Improved Nutrition (GAIN), have worked to better understand the adolescents' nutritional status, raise the profile of adolescent nutrition in Pakistan, and stimulate action on the topic. There has been significant progress in creating an enabling environment for adolescent nutrition, and Pakistan was one of the first countries to formalise and launch an Adolescent Nutrition Strategy and Operational Plan, in October 2020.

In this Working Paper, we describe the journey of policy formulation and strategy development for adolescent nutrition in Pakistan from 2016-2020, then present the current status of adolescent nutrition, alongside new findings around the attitudes, motivations and dietary practices of Pakistani adolescent girls and boys. Finally, we discuss possible strategic ways to strengthen multi-sectoral approaches to tackle adolescent malnutrition. Throughout the paper, we structure our examination by using the recently launched Innocenti Framework on Food Systems for Children and Adolescents (15).

\section{METHODOLOGY}

This Working Paper was developed through the review of policy documents and GAIN reports, reviewing key scientific literature, and conducting a series of semi-structured interviews with key stakeholders.

GAIN documents reviewed included published and unpublished reports, presentations, and other planning documents such as a baseline landscape analysis, programme policy reviews, and an action framework. Additionally, two studies of adolescents' motivation, behaviours, and consumption patterns, commissioned by GAIN and conducted by Ipsos and Quantum, were reviewed. The additional literature was collected via keyword searches using Google Scholar. We focused on adolescent nutrition, food insecurity, food safety, consumer behaviour, consumption patterns, and micronutrient deficiencies. Included literature was not older than five years, unless a study was of particular significance or if no recent data were available. Additional supporting information was collected through a grey literature search of reports, policy documents, and websites of departments of the Government of Pakistan and other agencies, including WHO, UNICEF, and global indexes.

Interviewees included representatives from the Government of Pakistan, partner organisations and national agencies, including the Ministry of National Health Services, Regulation and Coordination (MoNHSR\&C), Nutrition International, the National Fortification Alliance, UNICEF, WHO, the World Bank, and GAIN staff members and consultants. The goal of the interviews was to understand the challenges and opportunities in integrating and aligning adolescent nutrition policies and programmes, along with key lessons for future roadmaps. All interviews were conducted online and recorded to ensure that their content was available for 
later reference. All participants provided informed verbal consent to be interviewed and recorded.

\section{THE INNOCENTI FRAMEWORK}

The Innocenti Framework (Figure 1) on food systems for children and adolescents was developed by a consortium of technical experts led by UNICEF and GAIN to better understand the key drivers and determinants of children's and adolescents' diets, as well as the relationships between these factors. The ultimate aim of the framework is to highlight entry points for policymakers to design and implement policies and programmes to reshape food systems such that children and adolescents have access to nutritious, safe, affordable, and sustainable diets (15).

Figure 1. The Innocenti Framework on food systems for children and adolescents

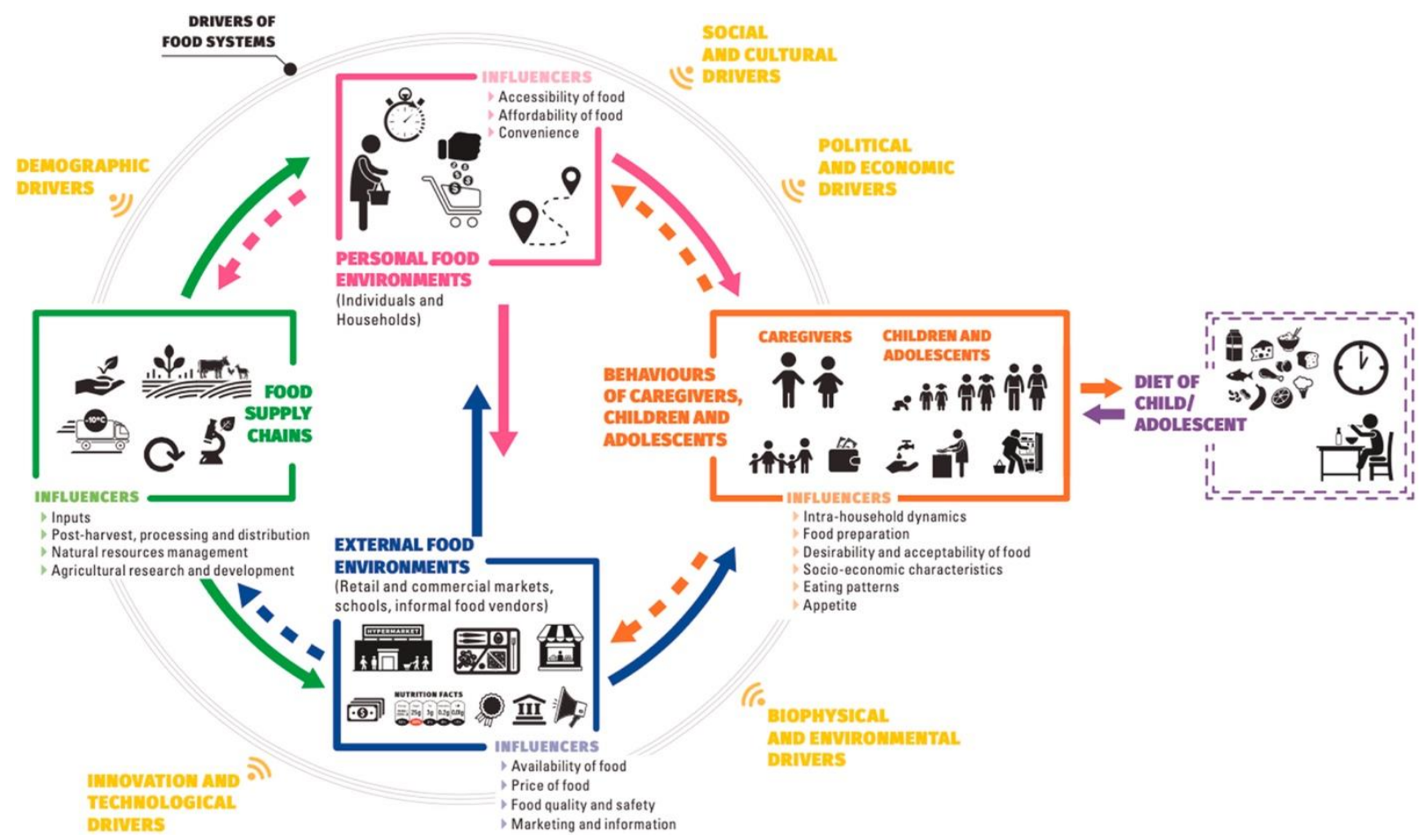

This paper considers the main parts of the food system in Pakistan, using this framework. Regarding the political and economic drivers of the food system, Part A of this paper reviews the progress Pakistan has made in strengthening the enabling environment for adolescent nutrition. Within this section, we highlight the recent key advances in data collection on outcomes and effective policy and programmatic measures for adolescent nutrition that helped inform the national strategy on adolescent nutrition. In Part $B$, we share recent findings on dietary behaviours and influencers of adolescents (displayed in orange in Figure 1), to examine motivations, life aspirations, dietary practices and consumption habits. Part $\mathrm{C}$ focuses on adolescents' food environment, specifically on the nutritional quality of readily available foods, their safety and on labelling and information. In the final sections, we reflect on how food system actors can build on the strategy and knowledge base now established for adolescent 
nutrition to improve young people's access to, and desire for, healthy and nutritious foods in Pakistan.

\section{PART A: BUILDING THE ENABLING ENVIRONMENT FOR ADOLESCENT NUTRITION}

Pakistan signed up to the Sustainable Development Goals in 2016 and became a member of the Global Scaling up Nutrition Movement in 2013, which helped elevate nutrition as a national priority. Multi-sectoral nutrition strategies and stunting reduction programmes were established, focusing on the first 1,000 days (from conception to 24 months). While it was generally recognised that young people in the 10-19-year age range did suffer from malnutrition, the nutrition issues faced by older children and adolescents were only partially addressed in policies or programmes. In 2016, the Nutrition Wing of MoNHSR\&C, alongside GAIN and partners, arranged a series of deliberations to map and understand the reality of adolescent nutrition in Pakistan.

\section{BRIDGING THE DATA AND POLICY GAPS ON ADOLESCENT NUTRITION}

First, GAIN and Aga Khan University conducted a situation analysis on the nutritional status of adolescent girls in Pakistan, which underscored significant data gaps on multiple variables, namely nutrition and food consumption behaviour, optimal modes of communication, and motivators of adolescent girls' health (14). This analysis found strong evidence that malnutrition and micronutrient deficiencies were of concern among girls in late adolescence (aged 15-19). It also found that very few programmes and interventions existed to tackle malnutrition among adolescent girls; those that did exist were limited in geographical coverage, not particularly integrated within health or educational or social protection programmes, and girls were primarily seen as 'women of reproductive age' (i.e., only of interest as part of the larger target population of mothers to be). This review also analysed data on eating habits collected via two food consumption surveys (conducted in 2014-15), finding that heavy consumption of staple foods such as bread/roti and rice was common, as was the consumption of tea and dairy. Other animal-source foods, pulses, fruits and vegetables were much less frequently consumed. These findings highlighted that the typical diet of adolescent girls is likely low in iron and other key micronutrients essential for growth and development. There were no findings related to prepackaged snack foods or drinks.

This research was presented at a national consultative meeting in May 2017, with the recommendation to develop guidelines on adolescent nutrition and supplementation. Following a series of consultations with key stakeholders from the public sector, development partners, and academia, the Nutrition Wing of MoNHSR\&C, with the support of WHO Pakistan, applied the WHO-led AA-HA! Framework to develop these guidelines for adolescents.

In August 2018, the first national platform dedicated to adolescent nutrition was established with the South Asia Food and Nutrition Security Initiative (SAFANSI). This platform, the National Technical Advisory and Advocacy Platform for Improved Adolescent Nutrition, is chaired by MoNHSR\&C, co-convened by GAIN and WHO, and includes representation from all regional provinces of Pakistan, as well as government agencies and departments, community support organisations, academia, and development partners. The Advocacy Platform aims to guide governments and partners to develop policies and programmes and select proven 
interventions to address adolescent malnutrition at national and sub-national levels using data, evidence, and learning from Pakistan. Through extensive multilateral collaboration with the World Bank, SAFANSI, and MoNHSR\&C, GAIN published two additional reports that built on the situation analysis and the guidelines and drew on this Advocacy Platform.

The second report, in 2018, examined nutritional status of adolescent girls and boys in Pakistan and highlighted the data gaps that made determining accurate patterns and trends in adolescent nutrition very challenging (16). This paper further investigated the scale of adolescent malnutrition, including amongst boys, who had largely been forgotten in prior assessments. The paper also explored dietary determinants among adolescents, concluding that there was very little data on dietary habits of adolescent boys and little understanding of their determinants. Key proximate determinants of adolescent malnutrition included poor dietary quality and infections during childhood; at the more distal level, they included poverty, food insecurity, low nutritional knowledge, poor access to health services, and intra-household food distribution issues.

The third paper, in 2019, undertook a critical policy analysis to propose 21 priority actions structured around seven themes (see Box 1) to address malnutrition in adolescents in Pakistan, based on prior $\mathrm{WHO}$ guidelines $(8,17)$. These actions are based on global and regional best practices to improve adolescent nutrition and provided direction to the PANS development process in the form of a 'Framework for Action'. The report was presented to the Advocacy Platform to review the actions and to harness further buy-in from policy and technical stakeholders.

\section{BOX 1: THE SEVEN 'FRAMEWORK FOR ACTION, POLICIES AND PROGRAMMES' THEMES}

Theme 1: Evidence based policy advocacy and sensitising government officials

Theme 2: Raising awareness on adolescent nutrition

Theme 3: Setting policy priorities for adolescent nutrition

Theme 4: Nutrition-specific interventions in the health sector

Theme 5: Nutrition-specific interventions in the non-health sectors

Theme 6: Scaling up for impact

Theme 7: Monitoring and evaluation, learning and accountability

These three seminal reports $(13,16,17)$ played a pivotal role in integrating data parameters on adolescents into the National Nutrition Survey (NNS) of 2018, which was one of the reports' recommendations. Prior to 2018, large-scale population-based surveys like the NNS and the Pakistan Demographic and Health Survey did not collect any precise information on adolescents, except for adolescent girls aged 15-19 as a part of the 'women of reproductive 
age' cohort. This data gap hindered effective programme and strategy design. The 2018 NNS was the first nationally representative survey to collect data on adolescent nutrition in Pakistan. The NNS 2018 was conducted by UNICEF and the Nutrition Wing of MoNHSR\&C and covered 145,000 adolescents (10-19 years old) representatively sampled across provinces and districts. Around two-thirds of the sample lived in rural areas and one third in urban areas. The findings, released in mid-2019, are briefly described in Box 2, below (18). 


\section{BOX 2. MALNUTRITION AMONG ADOLESCENT GIRLS AND BOYS (NNS 2018)}

NNS 2018 results indicate gender-based differences in both under- and overweight among adolescents in the country (18). One in five adolescent boys is underweight compared to one in eight adolescent girls (Figure 2). Conversely, more adolescent girls than adolescent boys are overweight. Obesity is also a public health issue among adolescents in Pakistan, with $7.7 \%$ of adolescent boys and $5.5 \%$ of adolescent girls affected. In all regions of the country, adolescent boys are more undernourished than adolescent girls (Figure 2). Of all population segments, rural boys are the most affected by undernutrition (20.8\%) and boys in the lowest economic segments are the most likely to be underweight (Table 2) (28.2\%). The NNS 2018 found that more than half (56.6\%) of adolescent girls in Pakistan are anaemic (with $0.9 \%$ being severely anaemic), with girls in rural areas more likely to be anaemic (58.1\%) than their counterparts in urban areas (54.2\%).

Figure 2. Nutritional status of adolescents in Pakistan by locality and gender

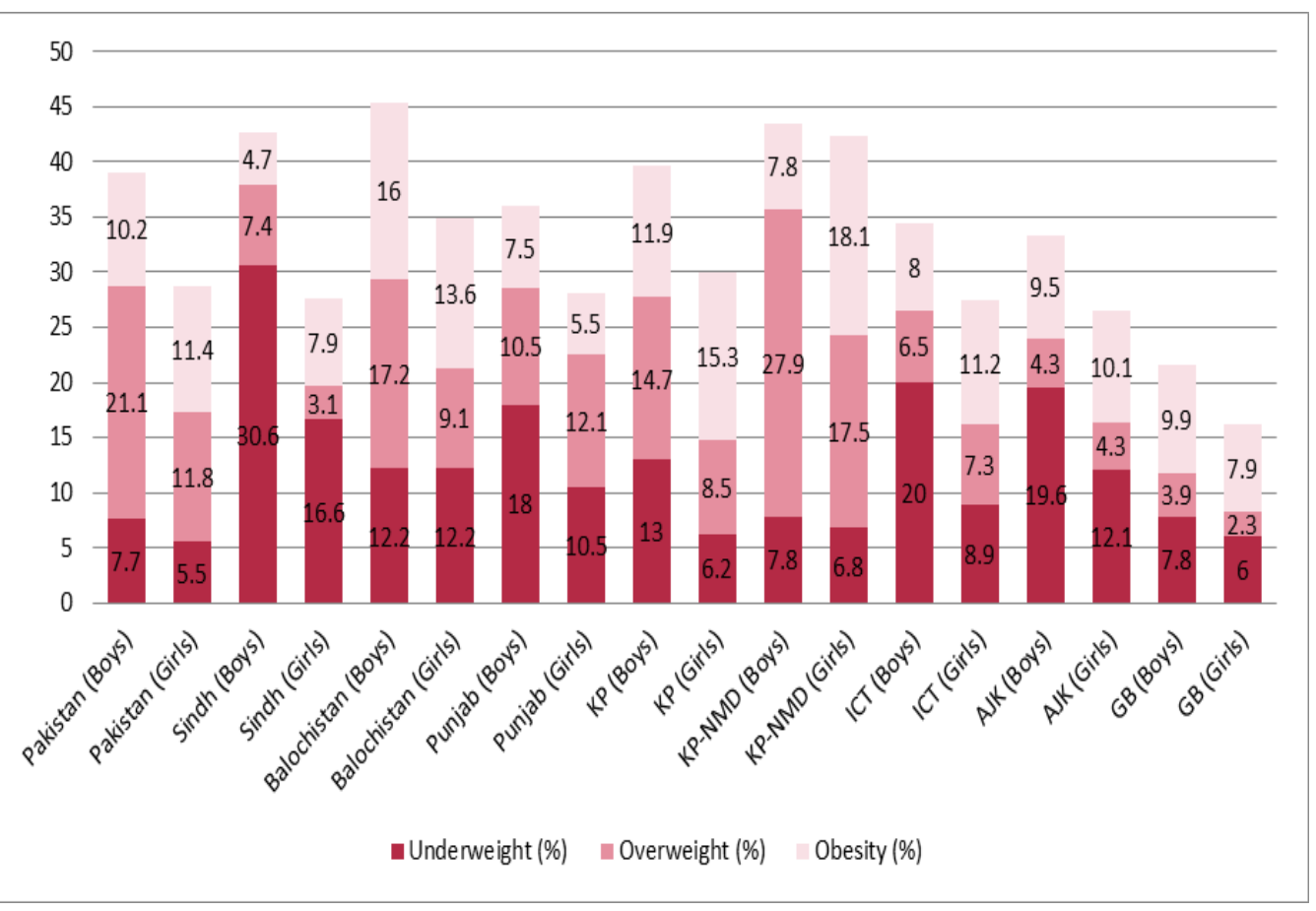

Table 1: Demographic and socio-economic differences in adolescent nutrition metrics (\%)

\begin{tabular}{|c|c|c|c|c|c|c|c|c|}
\hline & \multicolumn{4}{|c|}{ Girls } & \multicolumn{4}{c|}{ Boys } \\
\cline { 2 - 10 } & Underweight & Overweight & Obese & $\begin{array}{c}\text { Short } \\
\text { stature }\end{array}$ & Underweight & Overweight & Obese & $\begin{array}{c}\text { Short } \\
\text { stature }\end{array}$ \\
\hline Urban & 11.7 & 18.1 & 5.9 & 25.0 & 20.8 & 19.9 & 7.9 & 27.2 \\
\hline Rural & 11.8 & 16.1 & 5.2 & 30.4 & 21.3 & 16.4 & 7.5 & 34.7 \\
\hline Poor & 15.9 & 13.4 & 4.7 & 37.9 & 28.2 & 12.4 & 6.2 & 41.4 \\
\hline Wealthy & 10.5 & 20.8 & 6.9 & 19.1 & 17.7 & 21.6 & 7.8 & 19.8 \\
\hline No education & 10.8 & 13.9 & 4.3 & 32.9 & 24.7 & 17.8 & - & - \\
\hline High education & 7.9 & 16.3 & 5.3 & 18.1 & 15.7 & 21.1 & - & - \\
\hline
\end{tabular}




\section{DEVELOPMENT AND LAUNCH OF PAKISTAN'S ADOLESCENT NUTRITION STRATEGY}

Driven by political will, a stronger policy framework, and improved data (such as the milestone reports and disaggregated NNS 2018 results), the MoNHSR\&C, along with partners such as UNICEF, GAIN and WHO, embarked on a highly consultative process to develop the 'Pakistan Adolescent Nutrition Strategy and Operation Plan 2020-2025', formally launched in October 2020 (10). The launch was attended by representatives from each provincial government, who shared their commitment to the strategy. Objectives of the strategy are twofold: 1) to ensure that adolescent girls and boys benefit from supportive surroundings and adopt positive nutrition behaviours; and 2) to develop and implement evidence-based, multi-sectoral, highquality nutrition programmes and services at an appropriate scale to meet the needs of adolescent boys and girls. These objectives will be achieved via three strategic aims :1) the creation of a sustained enabling environment to address adolescent nutrition, 2) programmatic responses to adolescent nutrition across sectors, and 3) continued generation of evidence for guidance, learning, and accountability.

Up until now, programmatically, specific interventions targeting adolescents have been included in a few provinces (Sindh and Punjab, among others). However, these have been limited in terms of coverage (only implemented in a few districts), inclusiveness (little to no focus on adolescent boys), and scope (no focus on overweight/obesity) (10). For example, adolescent nutrition interventions are included in the Chief Minister's Three-Year Stunting Reduction Programme for Southern Districts of Punjab. The primary interventions include screening for malnutrition (mainly for adolescent girls), school-based water, sanitation, and hygiene (WASH) interventions, menstrual hygiene management, bi-annual deworming of adolescent girls, and the provision of micronutrient supplements (iron, folic acid, calcium, vitamin $\mathrm{D}$, and multivitamins) to adolescent girls. These interventions are primarily health system- and WASH-specific programmes and would need to be complemented by other sectors to attain their optimal impact on adolescents' diets and nutritional status.

Pakistan's Adolescent Nutrition Strategy (PANS) offers policy and programmatic mandates to provinces for multi-sectoral action towards adolescent nutrition, leveraging sectors such as agriculture and social protection. For example, agriculture extension workers will be trained in nutrition awareness and micronutrient distribution in under-served agricultural areas. Marginalised adolescents, including out-of-school adolescents, adolescents living with HIV, adolescents with disabilities, street children, ethnic minorities, transgender adolescents, adolescents incarcerated in prison and those in disaster or humanitarian situations, have been identified as needing special support. Recognising the importance of evidence-based policy advocacy and the need to sensitise government officials as advised in the 'Framework for Action', the federal government will initiate multi-sectoral dialogues on nutrition concerns in these groups and how to address them effectively. Social protection schemes, such as Ehsaas and the Benazir Income Support Programme, will be tasked to identify and reach the most vulnerable adolescents with cash transfers, food vouchers and other forms of support.

PANS includes the promotion of healthy diets by seeking prohibition of unhealthy food within schools and in their immediate vicinities and by offering nutritious meals at school canteens. PANS indicates that messages on healthy diets will be included in school curricula and teachers 
will be trained on nutrition awareness. The federal government also proposes offering conditional cash transfers and food vouchers to adolescents from poor households to increase school enrolment and reduce dropout rates. Another critical factor that influences adolescents' diets is the school environment, including mid-day meals served in schools. The Government of Punjab has been piloting the concept of providing lunch to students of 100 schools from November 2020 onwards (19). Eight schools are now covered, and the delivery organisation is on target to reach 100 within a year (20). The provision of nutrient-rich meals is expected to improve food insecurity and the educational performance of these students.

With such a positive enabling environment, Pakistan's government has shown commitment towards improving the lives and futures of adolescent boys and girls. These concerted efforts by MONHSR\&C and its partners, including UNICEF, GAIN, WHO, and others, have laid the foundation of bringing adolescent nutrition into the domains of policy, planning, and multisectoral programming. However, there is still a way to go to bring these policies beyond the health sector and truly engage adolescents to encourage them to change their attitudes and behaviours towards the foods they purchase and consume every day. For shifts in diets to happen, understanding the underlying determinants of food choices is critical, as is identifying specific food system actors who could support healthier diets. These topics are addressed in the next section.

\section{PART B: BEHAVIOUR AND INFLUENCERS OF ADOLESCENT DIETS}

In the Innocenti Framework, the behaviour of parents/caregivers and of adolescents themselves is the most proximate of the four determinants of adolescents' diets (displayed in orange in Figure 1). This determinant refers to intra-household dynamics, food preparation, eating practices, desirability and acceptability of food, and socio-economic characteristics. The Innocenti framework visualises this element as the point at which the external and personal food environments converge and as a buffer between food environments and adolescents' diets (15). This section explores the results of two studies into adolescents' behaviour and the motivations that underpin it.

\section{WHAT MATTERS TO ADOLESCENTS IN PAKISTAN AND WHAT ARE THEIR NUTRITION-RELATED MOTIVATIONS?}

A qualitative study, commissioned by GAIN and conducted by Quantum in 2019, focused on exploring insights that could shift adolescent behaviour and motivate them to consume nutritious food by analysing routines, dietary habits, and broader motivations in life (21). Nine, four-hour ethnographic studies with adolescents and their families (including home, school, and community life observations and tours) and nine ninety-minute group discussions were conducted. The adolescents lived in Karachi, Hafizabad, and Islamabad, and the group included a mix of both genders and in-school and out-of-school adolescents, divided into two age cohorts (10-14 and 15-19 years).

The results revealed that adolescents have a deep sense of respect and honour for their religions, families, communities, and the nation of Pakistan. Additionally, they see their growth and increased comprehension in education, equality, freedom, and social media and 
technology as new opportunities that can be used to secure a better life. The immense influence of local communities on the life of a family can have either a supportive or restrictive influence on the lives of adolescents. Respect within a community is a source of great pride for parents; however, adolescents feel that they face negative repercussions (such as disrespectful behaviour) and unnecessary criticism from community members, especially regarding the behaviour and honour of girls. Religion is also extremely important, reflected in the routines of adolescents and social practices of many families. Adolescents believe that personal growth and a well-paid job will increase their social status and economic stability. Similarly, parents want their children to receive a good education and strive for a well-respected career.

Even though a shift in families' attitudes towards adolescent girls (such as increased expectations related to educational attainment) is being observed in urban areas, there are still social pressures and limitations on how girls should dress and behave. For the most part, girls are not given much freedom to venture outside their homes. For adolescent girls in rural areas, preferential focus is given to marriage over education. More traditional families still do not see the benefit of educating their children (especially girls) beyond early adolescence. While there is an aspirational disposition among adolescents, socio-economic and cultural barriers hold them back from seizing new opportunities. Adolescents belonging to financially restricted families are often forced to drop out of school to start earning money, limiting their educational and career opportunities.

In terms of nutrition, adolescents are aware that a nutritious diet can help prevent disease, increase productivity, and contribute to the vitality and energy they need to achieve their desired goals. However, this is seen in very abstract terms ('good' vs 'bad' foods) and does not seem to strongly motivate them to consume more nutritious foods. There are also many structural barriers, such as food accessibility and availability, that limit healthy eating. Price hikes are a major cause of food insecurity, and healthy foods are perceived as more expensive. Furthermore, there is a lot of suspicion from both adolescents and parents about the overall purity, quality, and adulteration of food.

Canteens at educational institutions serve unhealthy items, such as junk foods and snacks, but adolescents' reluctance to bring a homemade lunch with them (rooted in a perception of this action as childish and indicative of parental dependency) leads them to eat whatever is available in the canteen. Eating outside and in restaurants also gives adolescents a sense of freedom, as their consumption options increase, although this experience is limited to older, urban adolescents. In addition, adolescents typically consume junk food with their peers, without their parents' approval. This sense of secrecy and camaraderie serves as a temptation, as it is a way to assert independence and strengthen the bond with their peers without any major consequences. Most adolescents in the sample do admit that their food consumption patterns need improvement.

Social media, family members, doctors, and teachers were the primary sources of adolescents' nutritional education. The study's results also indicate the increasingly detrimental role of the media in promoting unhealthy dietary habits through targeted marketing of sugar-sweetened beverages, specifically energy drinks, and unhealthy snacks. 

ADOLESCENTS IN PAKISTAN?

In 2020, GAIN and the market research agency, Ipsos, undertook a study analysing consumption, purchasing behaviours, and factors influencing dietary patterns to inform adolescent nutritional improvement programmes. The quantitative study, conducted from January-March 2020, used a sample of 2,331 girls and boys (12-19 years) from urban, semiurban, and rural areas across Pakistan. The qualitative study consisted of 12 online focus group discussions (FGDs) with 4-6 male and female 10-19-year-olds, across all socio-economic groups (except the highest one) from urban, semi-urban, and rural areas and were conducted in September 2020.

The results showed that adolescents typically ate regular meals with their families or in school. Snacking provided an opportunity for independent decision-making and rebellion, so snacks were typically nutrient-poor. The analysis of food category penetration, measured by selfreported consumption by adolescents in or out of the home within the past year, found that carbonated soft drinks (CSDs) and biscuits were the widely consumed foods at $75 \%$ penetration (Figure 3).

Figure 3. Food category penetration in adolescent diets

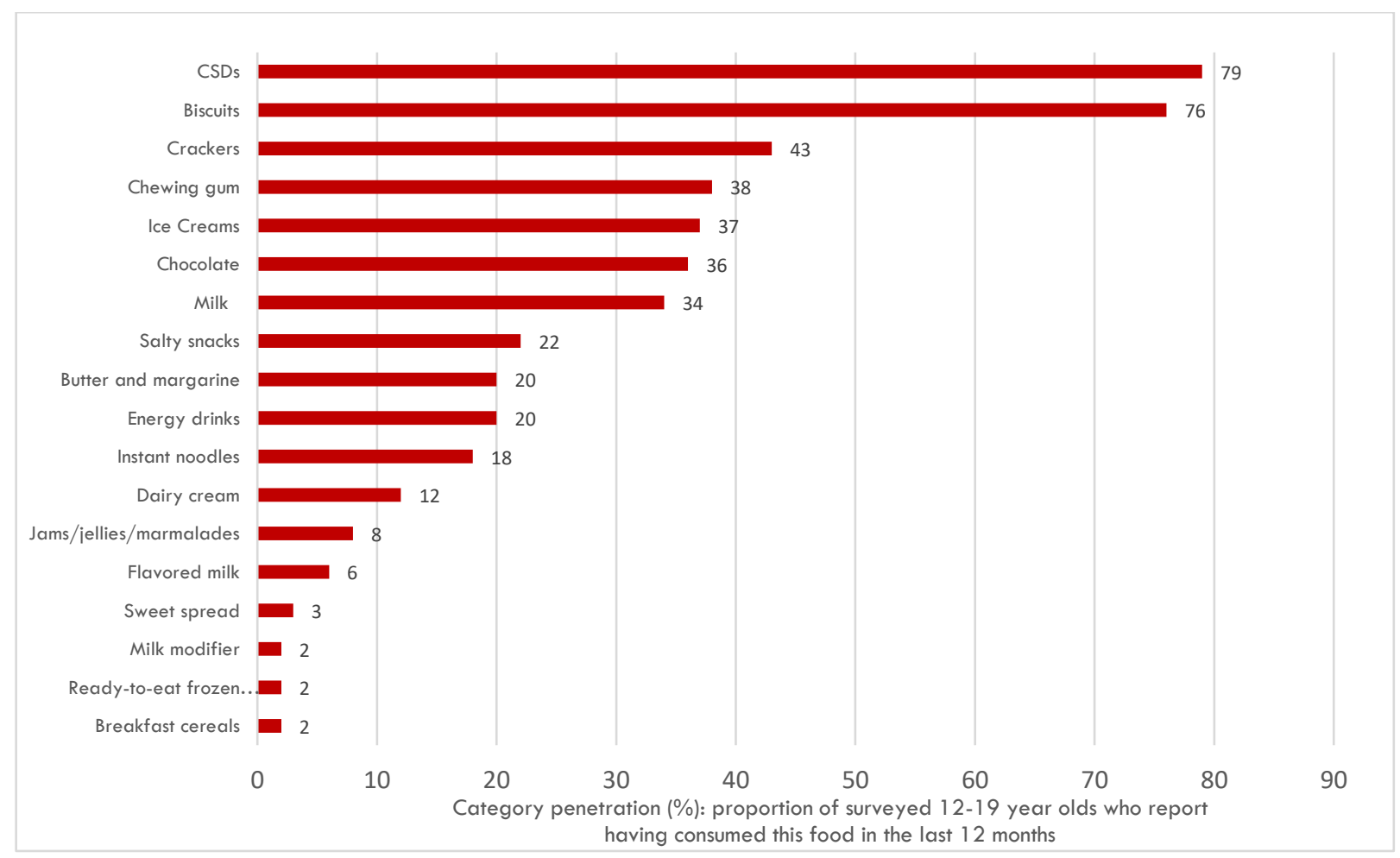

\section{CONSUMPTION OF DAIRY PRODUCTS}

Milk is the most commonly consumed form of dairy among Pakistani adolescents (34\%), most of which is used in milky, sweet tea. Adolescents do drink milk on its own, but those from lower socio-economic classes consider milk expensive and do not consume it on a daily basis. Adolescents from higher socio-economic classes have the option of fruit-flavoured milk, 
smoothies, and milkshakes, which are popular but high in sugars. After milk, ice cream is their most preferred dairy product, but is more accessible to urban than rural adolescents.

\section{CONSUMPTION OF SUGAR-SWEETENED DRINKS}

Consumption of sugar-sweetened drinks among adolescents is extremely high (Figure 4) and CSDs vastly exceed all other items, with 79\% penetration among adolescents and 2.3 CSDs consumed on average per week. CSDs are consumed more frequently by boys (2.5 times weekly) than girls (1.9 times weekly). Consumption is equal in urban and rural areas (2.2 drinks per week) and remains high even within the two lowest socio-economic groups (2.3 CSD drinks per week, closely followed by packaged juices at 2.1 times per week).

Figure 4. Weekly consumption (right-hand axis) and overall category penetration (left-hand axis) of sugary drinks among adolescents

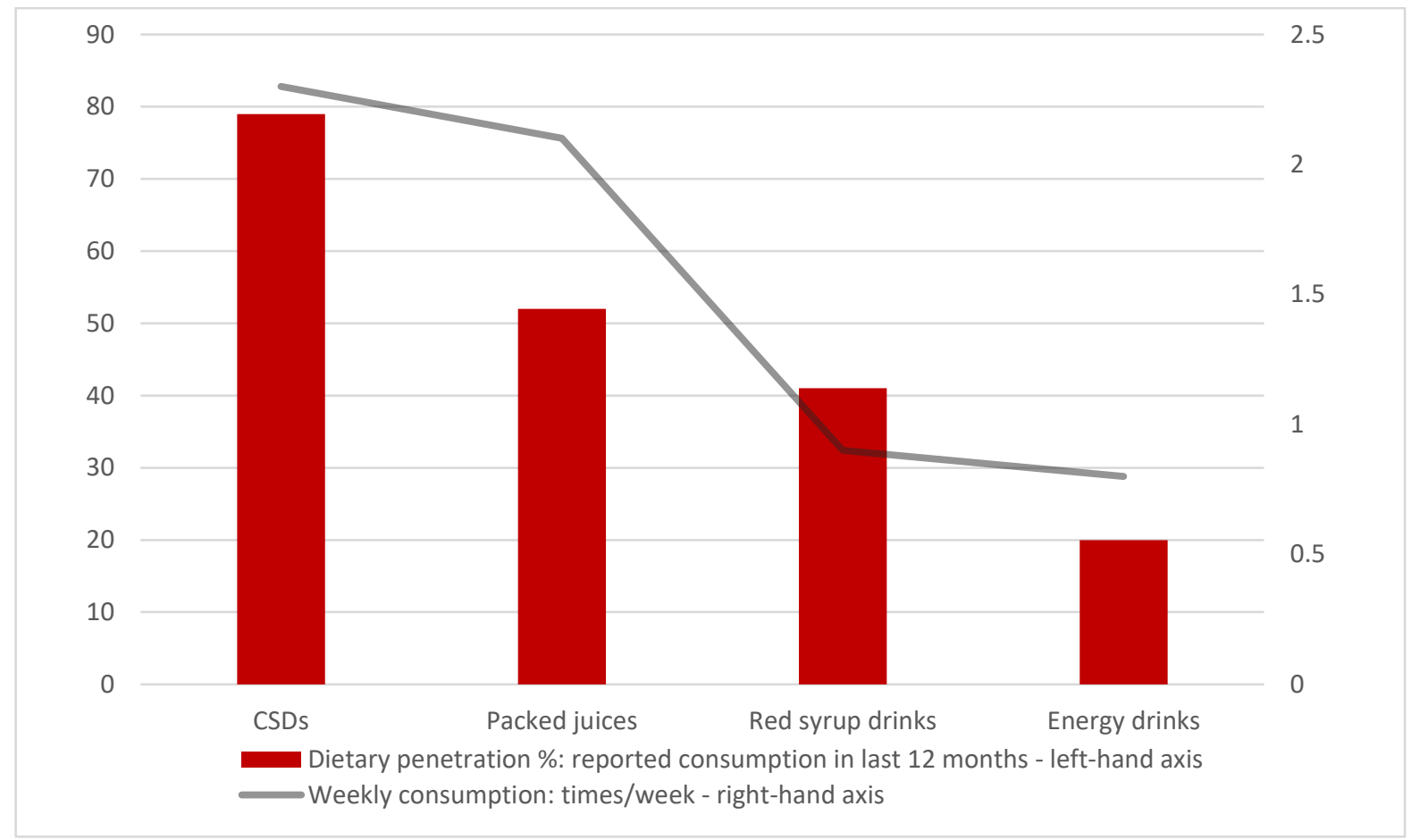

Affordability and accessibility issues mean that poorer adolescents and those from rural areas mostly consume homemade and traditional drinks (such as lassi and sardai), although these are considered to be 'uncool'. Penetration of energy drinks is higher among boys (27\%) than girls (7\%) as a result of targeted 'masculine' marketing (29), and strongly patterned by wealth. The success of CSDs across income groups, locations, and genders results from universal availability, exposure to heavy marketing efforts, and the taste and appeal of a CSD. Though there was awareness of their harmful effects on weight and body image, this concern was overpowered by the drinks' widespread availability, convenience and appeal. 


\section{SNACK FOODS}

Unsurprisingly, snacks are the most preferred food category among adolescents of all socioeconomic classes due to their easy accessibility, affordability and diversity of choice. Purchasing snacks also provides adolescents with a sense of freedom, as they can afford them with their own money. Adolescents from higher socio-economic classes and all those in urban areas mostly purchase international brands of snacks whereas limited availability and affordability means adolescents from rural areas purchase local brands. Weekly consumption of different types of snack foods is shown in Figure 5.

Figure 5. Weekly consumption of snack foods (times/week)

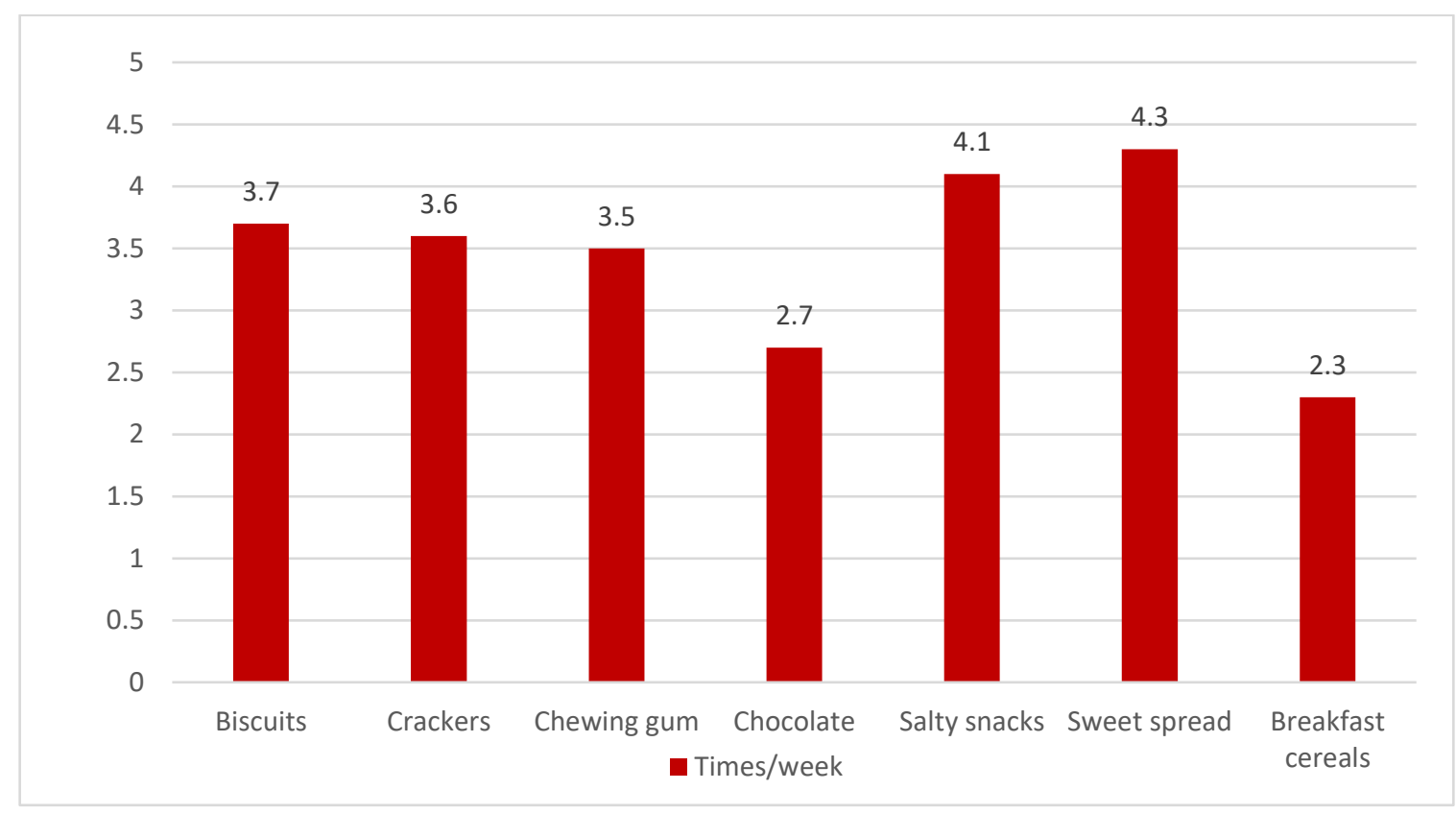

\section{INSTANT OR PACKAGED 'JUNK' FOODS}

Adolescents enjoy packaged foods, such as ready-made frozen foods (e.g., pizza, chicken nuggets) or instant noodles, as an alternative to the rice, lentils, and vegetables served in home-cooked meals. These items are convenient and readily available and are associated with 'luxury' and 'escape'. They are much more common in higher-income, urban areas, as rural adolescents find these foods inaccessible due to high prices (30).

\section{FRUITS AND VEGETABLES}

Adolescents were least enthusiastic about fruits and vegetables and saw eating them as an obligation imposed by their mothers. Despite their health benefits, fruits and vegetables contrast significantly with the varied options, extensive availability, and appeal of packaged unhealthy snacks. 


\section{PART C: FOOD ENVIRONMENTS}

Eating behaviours are shaped by both inter-personal and environmental factors. This section focuses on the Innocenti framework determinant of Food Environments (displayed in blue in Figure 1), which are the physical spaces in which consumers interact with food systems and encompass aspects such as availability, price, quality and safety, and marketing and regulation (15). A 2020 scoping review on the influence of external and personal food environment interventions on the diets of children and adolescents found very few published studies from LMICs. Most identified studies focused on school feeding programmes, educational programmes to improve nutritional knowledge in schools, or cash or food transfers in social protection systems. There was just one example of food and drink standards in LMICs, a smallscale intervention that sought to regulate tuck shop offerings in South Africa. An interesting experimental study from Guatemala found a significant taste preference for foods with a licensed character on the packaging (22). Cash transfer programmes in LMIC were found to improve dietary intakes, weight gain, and wasting but not stunting. One cash transfer programme combined with behaviour change communication found a positive impact on stunting in Bangladesh.

The results of the review indicate that evidence on how to improve child and adolescent diets in LMICs via the food environment is limited. Moreover, of those studies that do exist, most aim to target the personal food environment by improving purchasing power, largely to mitigate the negative impact of food insecurity on diets in many LMICs. As such, we focus our discussion of food environments for adolescents in Pakistan on three topics for which evidence exists: food insecurity (as a proxy for food access/affordability in the personal food environment) and food safety and marketing/labelling (both elements of the external food environment).

\section{PREVALENCE OF FOOD INSECURITY}

Pakistan has experienced overall growth in food production in recent years, which has led the country to produce more food than its population consumes. Several poverty alleviation strategies, social safety nets, and nutritional improvement programmes have also been introduced in recent years. However, many people in Pakistan still have poor access to food, partly due to low purchasing power and unstable economic circumstances (23). Indeed, two out of three households are unable to afford a nutritionally adequate diet (24). Economic and environmental shocks and systemic challenges (resulting in, for example, high postharvest losses) have further exacerbated the vulnerability of the population (25).

Pakistan is also considered at extreme risk of food insecurity, ranking as the $11^{\text {th }}$ most food insecure among 148 countries (25). The 2018 NNS found that 36.9\% of Pakistan's population faces food insecurity (based on the Household Food Insecurity Experience Scale, a selfreported measure of food-related behaviours and experiences, including low meal frequency and poor dietary diversity, associated with increasing difficulties in accessing food due to resource constraints (18)). A regional study conducted in rural Sindh on food insecurity among adolescents reported food insecurity among $52.4 \%$ of the adolescents compared to $39 \%$ of the households (26). Thirty percent of adolescents within food-secure households were food 
insecure. Adolescents were also more at risk of food insecurity than younger children; households with adolescents were twice as likely to report food insecurity than households with children under age five (27).

Food-insecure households often rely on low-cost (often less healthy) foods or adjust their intake by reducing portions and skipping meals. Adolescents in a food-insecure household often take cues from changes in household meals and voluntarily cut their portion sizes, a behaviour that often goes unnoticed by adults (27). In addition to impacting adolescents' nutrition, household food insecurity can also reduce academic performance and increase the school dropout rate among adolescents, resulting in social disadvantage during adulthood (27).

Despite this vulnerability, there is little emphasis on food security research among adolescents compared to that done on younger children and adults. Pakistan's first National Food Security Policy does aim to increase food accessibility among children but does not specify actions to improve adolescent food security. Food insecurity will likely be exacerbated by the COVID-19 pandemic and its impact on lifestyles, food systems, livelihoods and poverty (Box 3). 


\section{BOX 3. THE IMPACT OF COVID-19 ON ADOLESCENT NUTRITION IN PAKISTAN}

The global socio-economic crisis caused by the COVID-19 pandemic poses risks to the nutritional status of people globally, and adolescents in Pakistan are no exception. In Pakistan, specifically, an average change of $2.55 \%$ in the prices of 13 majorly consumed food items was observed in a four-month timespan (28). Such disruptions in food chains have affected households' access to food, reducing food intake and quality (29).

For adolescents, disruptions in educational facilities represented another major threat to food security, as Pakistan was one of the first countries in the world to institute widespread school closures (30). A study in July 2020 found that this closure of educational institutes resulted in a disorganised routine for adolescents, which encouraged habits such as latenight snacking and the overconsumption of nutrient-poor food, both of which increased substantially between February 2020-July 2020. As their sleeping schedules were also impacted, adolescents often missed important meals, such as breakfast. In addition, confinement has resulted in a decrease in physical activity as adolescents mostly turned to television, online gaming, and social media platforms for entertainment. However, preparation of food at home was reported to have increased by $28 \%$, which led to $32 \%$ of adolescents reporting an increased effort to consume healthy and nutritious meals, though $21 \%$ of adolescents also started making more desserts at home. Meanwhile, the shopping habits of adolescents also changed significantly, with a decrease in the number of shopping trips and the quantity and variety of items purchased (31).

As COVID-19 stretches the capacity of Pakistan's health system, millions of adolescents will go without basic healthcare and vaccines, and iron and folic acid supplementation, including for adolescent girls, has been interrupted. Additionally, monitoring of health and nutrition interventions was affected by restricted travel, and disruptions to nutrition programmes have rendered millions in dire need of nutrition support (32).

\section{FOOD SAFETY CHALLENGES}

The prevalence of foodborne illness in Pakistan is suspected to be very high, although there is no systematic surveillance or outbreak monitoring. Just $20 \%$ of people in Pakistan have access to clean drinking water; it is estimated that $50 \%$ of diseases and $40 \%$ of deaths occur due to polluted water (33). Harmful bacteria, aflatoxins, pesticide residues, heavy metal contaminants, and other adulterants in foods that cause harm to health have been found in many foods in many areas of Pakistan (34). Contaminated food supplies, inappropriate food storage, providing food from unhygienic sources, inadequate monitoring of food production, distribution and supply, and poor personal hygiene all critically affect food safety and increase the incidence of foodborne illness (35). For example, local milk vendors have been found guilty of mixing milk with contaminated water, harmful chemicals, detergent powder, and urea (36). A lack of outbreak monitoring and disease surveillance also hampers progress in tackling foodborne illness (34). 
According to the Pakistan Dietary Guidelines for Better Nutrition, food hygiene is not given any particular importance in the household or among commercial food providers (e.g., restaurants, street vendors) (37). In addition to strengthening the regulatory and enabling environment, educating consumers and producers on proper food handling and safety practices and motivating them to adopt them are essential to improve the safety of food in Pakistan and prevent foodborne disease outbreaks. Concerns about the safety, purity, and quality of informal and street vendors are suspected to motivate adolescents to reject their offerings in favour of pre-packaged snacks high in salt, fat and sugar. Similarly, shared (and suspected contaminated) drinking water facilities are rejected in favour of CSDs (38). It is important to understand how food safety concerns motivate and affect dietary choices among adolescents.

\section{MARKETING, NUTRITION LABELLING AND MISINFORMATION}

The evidence is unequivocal that marketing of unhealthy food and sugar-sweetened beverages is related to childhood obesity. Adolescents are specifically targeted by intense marketing strategies (such as the portrayal of popular figures for brand promotion or false health and nutrition claims displayed on food items) promoting unhealthy snacks and drinks (39). Their exposure to these marketing efforts is widespread - via TV, out-of-home media, retail merchandising, and more (40). Globally, regulation is either absent or tends to rest on ineffective industry-led self-regulatory pledges (39). Pakistan is no exception, and with a growing market for unhealthy foods and drinks, a regulatory framework on marketing and labelling is critical to halt the growth in preferences, discourage purchasing, and curb consumption.

Nutrition and health claims ${ }^{1}$ have the potential to make consumers more aware while making choices, increasing their understanding of specific nutrient-disease relationships, and are used by food manufacturers as marketing devices (41). Nutritional labelling provides consumers with the opportunity to choose healthier food options, while a lack of appropriate nutrient labels can lead to poorer food choices (41-43). Yet, despite the obvious benefits of nutritional labelling, Pakistan does not require nutritional content to be listed on product labels (44). A study conducted on nutrition labels of snacks in sixteen countries reported Pakistan to have the lowest number of mandatory nutrients listed; health and nutrient claims were also least commonly found on snack packets in the country (45). Analysing awareness of Pakistani consumers towards nutritional labelling, a small-scale study reported that expiration date is widely perceived as nutritional labelling and that consumers are largely unaware of the Guideline Daily Amount and essential nutrition facts (46). Whilst adolescents do have some agency in purchasing snacks and drinks, as the earlier studies here report, the majority of food is purchased by mothers for the wider household. Improving nutritional labelling has the potential to raise awareness among purchasers of food for adolescents, whether that is they themselves or their family (47).

${ }^{1}$ Nutrition and health claims include any form of short text describing the specific nutritional contents or health benefits of a food product. 


\section{DISCUSSION}

This examination adolescent nutrition in Pakistan has indicated several encouraging developments-as well as important gaps. First, PANS is an inspiring example of how political will can translate into effective policy and strategy and create a framework in which health and social system actors can understand the role they play in preventing and treating malnutrition. There is now a critical need for food system actors, specifically on the supply side, to better respond to this call to action and the impetus this enabling environment gives.

Second, the inclusion of adolescents as a distinct population in the NNS 2018 was a mammoth step forwards in furthering understanding of the scale of malnutrition in 10-19-year-olds. With $21 \%$ of boys and $12 \%$ of girls underweight, and $18 \%$ of boys and $17 \%$ of girls overweight or obese, Pakistan's adolescent population is experiencing a double burden of malnutrition. In addition, more than half of all adolescent Pakistani girls are anaemic. However, the NNS 2018 did leave gaps. It did not measure the prevalence of anaemia among boys and, given the high prevalence of malnutrition, it is likely that anaemia affects boys as well as girls. There are also no data on other micronutrient deficiencies among adolescent girls (these are covered collectively for 'women of reproductive age' i.e., mothers to be) or boys. As iron deficiency is the leading cause of morbidity and mortality in this age range worldwide, it is particularly critical to measure and monitor it in both boys and girls moving forward.

Building on the priorities highlighted in the 'Framework for Action' (17), an improved understanding of the effectiveness of existing adolescent nutrition programming in Pakistan is still needed, as is more research into the determinants of the country's poor adolescent nutrition, especially those related to gender, food insecurity, availability and affordability. Market surveys, such as the studies reported here, can play a role in filling these information gaps. The results presented here indicate that, although some adolescents have basic knowledge of the health impacts associated with poor dietary behaviour and excessive consumption of 'junk' food, they still eat and drink these foods for a variety of reasons. Key factors are diverse and include variety of choice, the ease with which the foods can be consumed as part of social gatherings and with friends, food accessibility and affordability, urbanisation, taste preference, and expression of independence. The purchase of these lowpriced 'junk' food items from pocket money also gives adolescents a sense of independence. The current selection is unhealthy, laden with salt, fat, and sugar, and a determinant of the poor nutritional outcomes we observe in this young population. Availability, accessibility, convenience, and variety, as well as social and cultural norms that stigmatise bringing homemade foods to social events, are key drivers of unhealthy snacking.

Whilst lacking the scientific rigour of longitudinal observational or experimental study designs, market surveys like these can provide rapid and topical insights into trends in purchasing, determinants, consumption and taste preferences. Food industry firms use such surveys to measure market potential and brand marketing success; the data presented in this paper show that their efforts in shaping adolescent diets in Pakistan have been remarkably successful despite widespread food insecurity. Policymakers and technical experts would thus do well to take note of industry techniques to understand consumption habits and determinants, as a complement to more scientific assessments. 
Indeed, the results presented here suggest several potential food systems interventions that could be used to improve adolescent nutrition. First, the packaged foods available to adolescents in their price range are ripe for innovation in nutritional composition and value. Similarly, nutritional labelling and marketing of packaged snacks offer an opportunity for supply side industry actors to innovate by offering more nutritious products and marketing to support their desirability. More importantly, they sound a call for enforcement and regulatory actors to safeguard consumers' nutritional needs. The high consumption of CSDs among all adolescents, regardless of gender, socio-economic status, or urban/rural location, but especially among boys, suggests the need for a strong fiscal policy measure (such as a tax) to make these unhealthy options less affordable to adolescents. Curbing the aggressive marketing of unhealthy foods and drinks to adolescents is a further key policy measure in which enforcement and regulatory actors could work alongside consumers to pressure industry to act. A desire to 'beat the heat' and quench thirst with refrigerated drinks is a key driver of CSD consumption amongst Pakistani adolescents. Industry can help meet this demand by reformulating with less sugars, sweeteners and stimulants, reducing portion sizes, and introducing new affordable, healthier products like nutritious, low-sugar flavoured milks or diluted lower-sugar fruit juices. Efforts to improve the safety and appeal of drinking water, especially in schools, are also critical.

Building on the PANS framework, school food environments and madrassahs are critical places in which food choices are made, social and cultural norms are established and enforced, and adolescents are relatively independent from their families. Actors in the food system and those in the health and social systems should work together to support schools in improving the food options, both in and around the school, available to their students. It should be easy to make a healthy choice when adolescents are at school. Finally, adolescents themselves could have socially transformative power if their collective interests in building healthier futures and healthier communities are harnessed. There is great potential for developing a youth movement for adolescent nutrition in Pakistan.

\section{CONCLUSION}

This paper has examined various aspects of adolescent nutrition in Pakistan and the food system that shapes it. Our examination of personal behaviours showed that adolescents in Pakistan do have some agency and independence in what, when and how they eat, and provided insights into their motivations, preferences, aspirations, and daily realities, which are crucial inputs when designing and deploying effective nutrition strategies. At the level of the enabling environment, the development of PANS indicated strong commitment to improving adolescent nutrition and illustrates the importance of multi-sectoral collaboration, especially across policy and technical stakeholders. The way this was shaped by major reports (from GAIN amongst other stakeholders) demonstrates the value of systematically identifying policy and data gaps to guide strategic and programmatic action.

With a food system in need of modernisation and a country facing serious economic constraints, tackling the high burden of undernourishment, overweight and obesity currently borne by adolescents in Pakistan is a huge challenge. Implementing the actions outlined in PANS will be important steps towards addressing this. To fully realise the potential of the food 
systems approach advocated by PANS, several additional steps are recommended to achieve a food system that benefits adolescents:

- Integrate adolescent nutrition-specific indicators in national monitoring and reporting frameworks to track progress, especially on micronutrient deficiencies and anaemia in boys.

- Systematically assess the determinants of malnutrition amongst adolescents (girls and boys), including how food environments shape their food preferences and food-related behaviours.

- Strengthen national and provincial-level multi-sectoral coordination focused on adolescents.

- Incentivise manufacturers to produce, and stimulate demand amongst adolescents for, a variety of healthier, cheaper and adequately marketed alternatives to unhealthy snacks and drinks, especially in and around schools.

- Consider fiscal interventions, like taxation, to discourage CSD consumption among adolescents.

- Implement and enforce existing food safety legislation, especially related to food inspection and regulation.

- Implement labelling regulations that incentivise manufacturers to develop products that promote public health and help consumers follow dietary recommendations. 


\section{REFERENCES}

1. Sheehan $P$, et al. Building the foundations for sustainable development: a case for global investment in the capabilities of adolescents. The Lancet Regional Health. 2017; 390: 1792-1806.

2. United Nations Department of Economic and Social Affairs. 2019 Revision of World Population Prospects. Available from: https://population.un.org/wpp/ [Accessed 19th October 2020].

3. Bonnie RJ, Backes EP. The Promise of Adolescence: Realizing Opportunity for All Youth. Washington, DC: The National Academies Press; 2019.

4. Galler JR, Koethe JR, Yolken RH. Neurodevelopment: The Impact of Nutrition and Inflammation During Adolescence in Low-Resource Settings. Pediatrics 2017; 139.

5. Prentice $A M$, et al. Critical windows for nutritional interventions against stunting. The American Journal of Clinical Nutrition. 2013; 97: 911-918.

6. Global Nutrition Report. Nourishing the SDGs. Bristol: Development Initiatives Poverty Research Ltd; 2017.

7. Food and Agriculture Organization, Ministry of Planning Development and Reform. Pakistan Dietary Guidelines for Better Nutrition. 2018.

8. World Health Organization. Guideline: Implementing effective actions for improving adolescent nutrition. Geneva: World Health Organization. 2018.

9. Usman A, Jalil A, Amjad A. Food, City and Young Globavores: Perceptions of Pakistani Urban Youth about Food Choices and Healthy Eating. Anthropologist. 2017; 27(1-3): 143-151.

10. Ministry of National Health Services Nutrition Wing, Regulations and Coordination, Global Alliance for Improved Nutrition, World Health Organization, UNICEF. Pakistan Adolescent Nutrition Strategy and Operational Plan. 2020.

11. Saghaian S, Mohammadi H. Factors Affecting Frequency of Fast-Food Consumption. Journal of Food Distribution Research. 2018; 49(1).

12. Campisi SC, et al. Later puberty onset among chronically undernourished adolescents living in a Karachi slum, Pakistan. Acta Paediatrica. 2019; 109(5): 1019-1025.

13. van Liere M, et al. Embodying the Future: How to Improve the Nutrition Status of Adolescent Girls in Pakistan. Geneva: Global Alliance for Improved Nutrition. 2017.

14. UNICEF. COVID-19, Climate Change \& Environmental Degradation: Key Asks for Public Sector Partners. 2020.

15. Raza A, et al. Conceptual framework of food systems for children and adolescents. Global Food Security. 2020; 27(100436).

16. Global Alliance for Improved Nutrition (GAIN). Technical Report: Review of evidence on the nutritional status of adolescent girls and boys in Pakistan. Geneva: GAIN; 2018. 
Available from: https://www.gainhealth.org/resources/reports-andpublications/review-evidence-nutrition-status-adolescents-girls-and-boys-pakistan

17. Badar A, Rasool F, Pasha QM, Beal T, Tumilowicz A, Rabeneck S. Framework for Action - Policies and Programmes. A policy paper on Adolescent Nutrition in Pakistan: A Framework for Action, Policies, and Programmes. 2019. Available from: https://www.gainhealth.org/sites/default/files/publications/documents/world-banksafansi-govt-of-pakistan-gain-framework-for-action-programs-and-policies-a-policypaper-on-adolescent-nutrition-in-pakistan.pdf

18. UNICEF, Nutrition Wing Ministry of National Health Services, Regulation and Coordination (MoNHSR\&C). National Nutrition Survey; 2018.

19. Children of 100 primary schools in Punjab to receive a free meal daily. The News International. Available from: https://www.thenews.com.pk/latest/739449-children-of100-primary-schools-in-punjab-to-receive-a-free-meal-daily. [Accessed 5th November 2020].

20. Classroom Hunger affects Children's ability to Learn and Grow. The Allah Walay Trust. Available from https://allahwalaytrust.org.pk/school-meal-project/

21. The Global Alliance for Improved Nutrition (GAIN) Pakistan and Quantum Understanding Adolescent Motivations in Pakistan; 2019 (unpublished)

22. Letona $P$ et al. Effects of licensed characters on children's taste and snack preferences in Guatemala, a low/middle income country. Int. J. Obesity 38 (2014), pp. 1466-1469,

23. USAID. Food Assistance Fact Sheet Pakistan; 2020.

24. Ministry of Planning, Development and Reform and World Food Programme. Minimum Cost of the Diet; 2016. Available from: https://reliefweb.int/sites/reliefweb.int/files/resources/mcod pakistan july 2016.pdf

25. Adeeba I, Khalid M, Ahmad E. Food Insecurity in Pakistan: A Region Wise Analysis of Trends. 2018, Pakistan. Institute of Development Economics. 2018; 157.

26. Sheikh $S$, et al. Adolescent food insecurity in rural Sindh, Pakistan: a cross-sectional survey. BMC Nutrition. 2020; 6(17).

27. Dush JL. Adolescent food insecurity: A review of contextual and behavioral factors. Public Health Nursing. 2020; 37: 327.

28. World Food Programme. Rapid Assessment - Possible Impact of the Novel Coronavirus Pandemic (COVID-19) on Livelihoods, Food Security, Nutrition and Agricultural Supply Chain in Pakistan; 2020.

29. Headey D, et al. Impacts of COVID-19 on childhood malnutrition and nutrition-related mortality. The Lancet. 2020; 396(10250): 519-521.

30. Geven K, Hasan A. Learning Losses in Pakistan due to COVID-19 School Closures: A Technical Note on Simulation Results. Washington D.C.: World Bank; 2020. 
31. Ipsos Pakistan. Post COVID-19 Understanding Consumer Behaviours Shifts in Pakistan. July 2020 available at https://www.ipsos.com/sites/default/files/202007/post covid handbook.pdf

32. Global Alliance for Improved Nutrition. Impact of COVID-19 on Food Systems: A Situation Report, Edition 4. November 23, 2020; 2020.

33. Daud, M.K, et al. Drinking Water Quality Status and Contamination in Pakistan. BioMed Research International 2017.

34. Rauf $M$, et al. Pakistan food safety challenges. International Journal of Biosciences, 2020; 17(2): 17-28.

35. Akhtar S, et al. Food safety conundrum: a Pakistan's scenario. Quality Assurance and Safety of Crops \& Foods, 2015; 7(4): 559-567.

36. Lateef M, Faraz A, Mustafa MI. Detection of adulterants and chemical composition of milk supplied to canteens of various hospitals in Faisalabad city. Pakistan Journal of Nutrition. 2009; 9: 139-142.

37. Food and Agriculture Organization and Ministry of Planning Development and Reform. Pakistan Dietary Guidelines for Better Nutrition. 2018.

38. Datta BK, Husain MJ. Carbonating the household diet: a Pakistani tale. Public Health Nutrition. 2020; 23(9):1629-1637.

39. Tatlow-Golden, M and Garde, A. Digital food marketing to children: Exploitation, surveillance and rights violations. Global Food Security, Volume 27, 2020

40. Li L, et al. Fast food consumption among young adolescents aged $12-15$ years in 54 low- and middle-income countries. Global Health Action. 2020; 13(1).

41. Annunziata A, Mariani A. Do Consumers Care about Nutrition and Health Claims? Some Evidence from Italy. Nutrients. 2019; 11(11).

42. Ikonen I, et al. Consumer effects of front-of-package nutrition labeling: an interdisciplinary meta-analysis. Journal of the Academy of Marketing Science. 48: 360383.

43. Bablani $L$, et al. The impact of voluntary front-of-pack nutrition labelling on packaged food reformulation: A difference-in-differences analysis of the Australasian Health Star Rating scheme. PLoS Medicine, 2020; 17(11): 1003427.

44. Khalid SMN. Food Labeling Regulations in South Asian Association for Regional Cooperation (SAARC) Countries: Benefits, Challenges and Implications. Turkish Journal of Agriculture - Food Science and Technology. 2015; 3(4): 196-203.

45. Mayhew AJ, et al. Nutrition labelling, marketing techniques, nutrition claims and health claims on chip and biscuit packages from sixteen countries. Public Health Nutrition. 2016; 19(6): 998-1007.

46. Zaidi SHA, Muhammad B. Awareness of Pakistani Consumers towards Nutritional Labeling on Product Packaging in Terms of Buying Behavior. International Journal of Business and Social Science. 2012; 3(16): 97-103. 
47. Talagala IA, Arambepola C. Use of food labels by adolescents to make healthier choices on snacks: a cross-sectional study from Sri Lanka. BMC Public Health. 2016; 16(739). 